\title{
COOPERATIVE PROGRAM TO ANALYZE HEAT TRANSPORT AT HIGH BETA IN DIII-D
}

Contract DE-FG03-89ER51116

\author{
T. Kenneth Fowler and Taina K. Kurki-Suonio, Editors \\ University of California, Berkeley \\ Department of Nuclear Engineering
}

\begin{abstract}
DISCLAIMER
This report was prepared as an account of work sponsored by an agency of the United States Government. Neither the United States Giovernment nor any agency thereof, nor any of their employees, makes any warranty, express or implied, or assumes any legal liability or responsibility for the accuracy, completeness, or usefulness of any information, apparatus, product, or process disclosed, or represents that its use would not infringe privately owned rights. Reference herein to any specific commercial product, proce ss, or service by trade name, trademark, manufacturer, or otherwise does not necessarily constitute or imply its endorsement, recommendation, or favoring by the United States Government or any agency thereof. The views and opinions of authors expressed herein do not necessarily state or reflect those of the United States Government or any agency thereof.
\end{abstract}

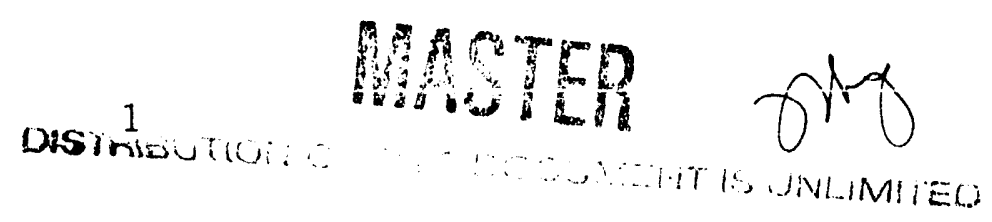




\title{
CONTRIBUTORS
}

\author{
Alain Brizard ${ }^{1}$ \\ Daniel Finkenthal ${ }^{2}$ \\ Joseph Fitzpatrick ${ }^{3}$ \\ T. Kenneth Fowler ${ }^{4}$ \\ Daniel Hua ${ }^{2}$ \\ Taina Kurki-Suonio ${ }^{5}$ \\ Bijal Modi ${ }^{2}$ \\ Quang Nguyen ${ }^{3}$ \\ Xueqiao Q. $\mathrm{Xu}^{6}$
}

1 Lawrence Berkeley Laboratory

2 Graduate students supported by the grant

${ }^{3}$ DOE Graduate Fellowships

4 Principal Investigator

5 Assistant Research Engineer

${ }^{6}$ Department of Electrical Engineering and Computer Science 


\section{CONTENTS}

1. Ion Temperature Analysis

-data analysis

-'cook book'

-code development

-effect of ELMs on Ion Temperatiure Profile

2. Thermal Transport in DIII-D

-spatially and temporally resolved $\chi_{e}$ and $\chi_{i}$ across $\mathrm{L}-\mathrm{H}$ transition -evolution of plasma profiles across the transition

-correlating turbulence suppression and improved confinement

3. Particle Transport in DIII-D

-thermal pinch as an explanation for the shape of $\mathrm{H}$-mode $\mathrm{n}_{e}$ profile -upgrade of ONETWO to include drift-wave produced thermal pinch -comparison of ONETWO runs to experiments

4. Divertor Design

5. Non-Circular Geometry

6. TAE-mode

7. Appendix A

-Publication List

-Abstracțs 


\section{COOPERATIVE PROGRAM TO ANALYZE HEAT TRANSPORT AT HIGH BETA IN DIII-D}

This is the final report for work funded under U.S. Department of Energy Contract DE-FG03-89ER51116, November 11, 1988 through November 10, 1991.

Our work is now fully integrated into the ongoing efforts at GA to help expedite progress in the fundamental understanding of transport phenomena in tokamaks. Results to date, outlined below, have been reported in 31 journal publications, laboratory reports and meeting presentations listed herein. Close communication with GA staff members is maintained by computer linkage and by frequent trips to San Diego. Dr. Gary Porter, who leads the Livermore DIII-D team, is a regular participant in our group meetings in Berkeley.

The main contribution of the Berkeley group to date has concerned ion temperature profilc data reduction and transport analysis using this data. In addition, our graduate students have worked on fundamental aspects of transport theory, under the guidance of the Principal Investigator, to prepare them for productive participation in the DIII-D program. One student has coauthored a paper on divertor design, a subject of increasing urgency for ITER and an area of increasing importance in the DIII-D program. Finally, work has been completed on modifying thermodynamic and ITG theories for the non-circular geometry of DIII-D, with some interesting consequences for DIII-D transport analysis.

The following is a brief summary of this work. More details are included in the publication abstracts given in Appendix A.

\section{ION TEMPERATURE ANALYSIS}

Among the data essential for transport analysis in DIII-D, the ion temperature profile analysis has proven to be most difficult to automate, and in fact this analysis has generally required the time-consuming attention of the experimentalists. With encouragement from the GA management, the Berkeley group made a commitment to assist GA in sharing the burden of this analysis work and implementing improvements in the process.

The ion temperature measurements at DIII-D are based on spectroscopic observations of the signal from the Charge Exchange Recombination (CER-process) when the neutral beams are present in the plasma. By now there are 32 chords (16 looking in vertically at the plasma, and 16 looking in the horizontal plane) looking at different locations in the plasma along the beam path. The spectral signal corresponding to each of the chords is analyzed separately, and the analysis is complicated because the measured spectrum has contributions not only from the CER-process with the beam, but also from other atomic processes near the edge of the plasma. The relative intensities of these components can vary greatly, and in unfavorable cases, the lines emitted at the edge can interfere severely with 
the temperature measurement at the beam location. The analysis is further complicated by the presence of fainter impurity lines from atomic transitions of mainly carbon, nickel and oxygen. Due to the complexity of the data, great care is required in the analysis and refits of the data are often required. Evaluation of the ion temperature fit is made between the iterations. This evaluation is based on experience that one gains only after relatively long hands-on experience with the analysis. The recent addition of new chords to obtain information at the edge of the plasma makes the procedure even more time consuming.

Considerable progress has been made along two lines. First, beginning with the assignment of one of our graduate students (D. Finkenthal) to San Diego for an extended period in the summer of 1989 , the Berkeley group began to share the workload of reducing the ion temperature profile data by the labor-intensive methods then in place. Dr. Taina Kurki-Suonio, who joined our group as a postdoctoral fellow in May 1989, continued some of this work. This load-sharing enabled Dr. R. Groebner at GA, who had previously been almost entirely responsible for producing the profiles, to devote more time to improving the procedure. Mr. Finkenthal also compiled the standard procedure into a little handbook that would enable novices to get started in the $T_{i}$-analysis. Secondly, a graduate student expert in computer programming (B. Modi) spent the summer of 1990 at GA, during which he assisted the GA group in implementing various planned improvements to the analysis code as well as some of his own ideas. Major contributions during the summer were to speed up the error analysis algorithm substantially and to examine the software carefully to make speed improvements where possible. He is now contributing toward the ultimate automation of the ion temperature profile analysis by installing a new least squares minimizing algorithm in the code. This work, when completed, will be the main topic of Mr. Modi's Master's Thesis.

Dr. Kurki-Suonio also did a study on the effect of ELMs on the ion temperature at various locations in the plasma (with major radii $2.29 \mathrm{~m}, 2.26 \mathrm{~m}$ and $2.13 \mathrm{~m}$ ). This study covered five ELMs and a clear drop in the ion temperature was observed at all locations across each of the ELMs.

\section{THERMAL TRANSPORT IN DIII-D}

Understanding why and how the H-mode exhibits improved particle and thermal confinement is essential before a commercial reactor can be designed. In close collaboration with Drs. Groebner and Burrell at G.A, Dr. Kurki-Suonio has studied the details of thermal and angular momentum transport in DIII-D plasma across the L-H transition. Based on plasma profiles obtained from hot ion $\mathrm{H}$-mode discharges on DIII-D, the transport analysis showed that the improvement in confinement takes place all across the plasma cross-section (and not just at the edge). The improvement is not instantaneous. A drop in the diffusivities was observed to begin within $30 \mathrm{~ms}$ following the transition and the improvement continues until at least $80 \mathrm{~ms}$ after the transition. The dominant improvement is in the electron channel. The nature of changes in the electron density is similar to the changes in diffusivities suggesting that the density gradient length is a critical quantity in 
the L-H transition. Experimental data is compared to a model for ITG mode. Stabilization of the ITG-mode does not seem to take place in the L-H transition, but the plasma might be marginally stable against the mode. The preliminary results were presented at the Division of Plasma Physics meeting, November 1990, and a complete report has been accepted for publication in Nuclear Fusion.

As a continuation for this study we are currently analyzing a series of more recent shots that have fluctuation measurements. Comparing the temporal behavior of the fluctuations and the transport coefficients in the bulk of the plasma, it appears that the fluctuations are suppressed in the same time scale as the reduction in transport is observed, and that the zone where the fluctuation suppression is observed overlaps with the region where the transport coefficients are reduced the most. The preliminary results of this study are to presented in the 1991 APS meeting in Tampa, Florida.

\section{PARTICLE TRANSPORT ANALYSIS IN DIII-D}

In collaboration with GA and LLNL, we have initiated a study to look for the dynamics responsible for producing the flat, shouldered electron density profiles observed in DIIID H-mode plasmas. In a previous transport study it has been shown that a significant inward pinch terrin was required to numerically reproduce the observed density profile. In this study the particle diffusion coefficient and the pinch velocity were empirically adjusted to obtain consistency between the calculated and experimental density profiles. We are taking this study further by including theoretical models. In particular, we are looking at the thermoelectric-type effects as the source for the inward pinch. Dr. Kurki-Suonio has implemented a model (by $R$. Waltz) for a drift-wave produced pinch into the ONETWO code and preliminary runs have indicated that the particle flux due to this pinch is a significant fraction of the total flux. For a set of more recent discharges in DIII-D, the analysis of the soft X-ray signal by Dr. Gary Porter (LLNL) further suggests the presence of a particle pinch. To verify this we are comparing the experimental profiles to the ones obtained from the upgraded version of ONETWO, in which the value for the pinch velocity now comes from a theoretical model.

\section{DIVERTOR DESIGN}

A graduate student (Q. Nguyen) has begun work on designs to reduce the divertor heat flux by employing poloidal coils to spread the scrape-off layer field lines over a wider area before they intersect the divertor plates. First results were presented at the Division of Plasma Physics meeting, November 1990, and a report has been prepared, co-authored with Dr. Fowler, and Dr. Stambaugh at GA.

The method appears promising and may contribute to improved confinement by pro- 
viding design flexibility to simultaneously handle the heat flux together with appropriate baffling to contain and pump gases and impurities that would otherwise spoil the confinement.

\section{NON-CIRCULAR GEOMETRY}

A paper has been accepted for publication that applies thermodynamic methods to calculate non-linear upper bounds on fluctuation levels and growth constants for electrostatic drift waves. The aim of this work was to try to understand the origin of the empiricallyobserved strong dependence of the global energy confinement time on the current, whereas heretofore linear theories and diffusion estimates based on them depend mainly on the toroidal field (gyro-Bohm scaling). As was noted in our original grant proposal, this is a central issue in the design of future tokamaks (including BPX and ITER), especially in the strong reliance in these designs on elongation of the plasma cross section as a means of increasing the current; and DIII-D, operating at high beta and an elongation $\kappa \geq 2$, is uniquely suited to exploring this issue.

When it was found that the thermodynamic theory, of wide validity even for complex geometry, failed to disclose significant current or geometry dependence, a detailed study was undertaken of a particular instability, the ITG mode, by adapting an existing linearized stability code to the non-circular geometry appropriate to DIII-D. It was found that the growth constant $\gamma$ and a mixing-length extimate of the ion heat conduction, $\chi \sim \gamma / k^{2}$, do exhibit a strong dependence on elongation and current, and this discrepancy from the thermodynamic bound was traced to the fact that the tokamak would be expected to hover near marginal stability for the ITG mode (which exhibits a critical gradient) whereas the thermodynamic estimate assumes that the system is far from marginal stability. Moreover, while the ITG theory is too sensitive to details to claim agreement or disagreement with the DIII-D data, a model for $\chi_{i}$ based on the theory does agree qualitatively with features of the data not easily explained by other theories. This is illustrated in Fig. 1, which plots $\chi_{i}$ derived by solving the model heat flow equation versus experimental values from a hot-ion H-mode shot in DIII-D. Note that, because of the critical gradient effect, the model $\chi_{i}$ increases at large $\rho=\mathrm{r} / \mathrm{a}$ (like the experiment) despite a proportionality to $\mathrm{T}_{i}^{3 / 2}$ as in other gyro-Bohm formulas. Also, the $\mathrm{T}_{i}(\rho)$ profile is relatively insensitive to the heating profile (similar to the 'heat pinch').

The thermodynamic theory, based on gyrokinetic equations, was carried out by a graduate student (D. Hua) in collaboration with the Principal Investigator and Dr. Alain Brizard, a postdoctoral fellow at LBL, and the linear ITG analysis was carried out by Mr. Hua and another post-doctoral fellow on campus, Dr. Xueqiao Q. Xu, who had worked on this problem at UCSD prior to coming to Berkeley. This work will be a part of Mr. Hua's $\mathrm{PhD}$ thesis, soon to be completed. 
Figure 1.

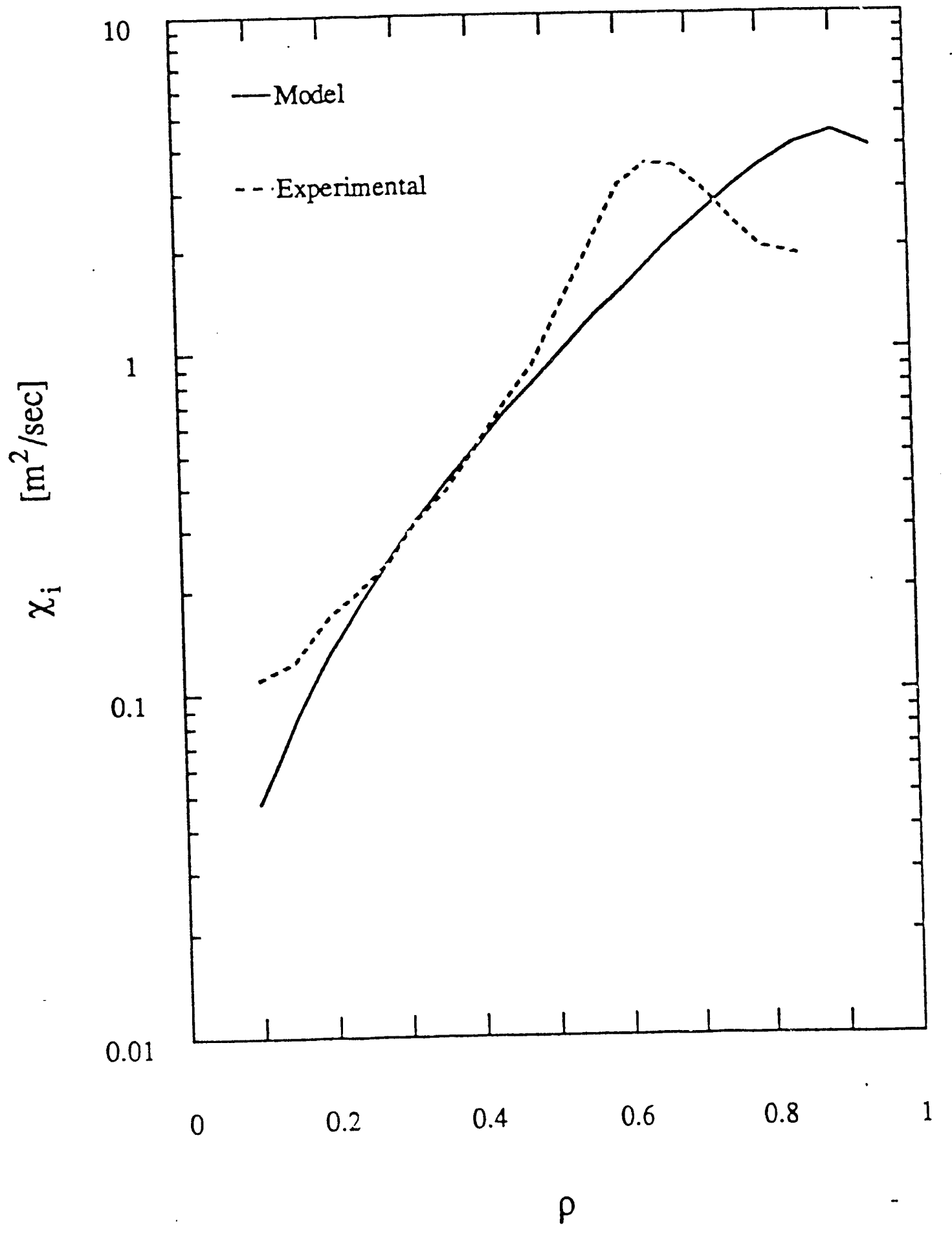




\section{TAE MODE}

A mixing-length transport model has been used to estimate alpha transport by the TAE mode and its effects on reaching ignition. The transport coefficient is taken to be $\mathrm{D}=\gamma / k^{2}$ with $\gamma$ given in the paper of $\mathrm{Fu}$ and Van Damm. In this formula, $\mathrm{k}$ is chosen to be that value which maximizes $\mathrm{D}$, namely $\mathrm{kr}_{L \alpha} \sim 5$ in the core ( $\operatorname{similar}$ to $\mathrm{kr}_{L}$ in DIII-D experiments in which neutral beams excite TAE-like modes). It is found that transport self-limits $\mathrm{D}$ by limiting the accumulation of alpha density $\mathrm{n}_{\alpha}, \gamma$ (and hence D) being a function of $\mathrm{n}_{\alpha}$. Nonetheless, for this model, the transport rate is sufficient to spread the alphas enough to reduce their effective heating power in the core to only $10-20 \%$ of what it would be in the absence of TAE transport. The actual transport would be less if only a select group of alphas - those resonant with the waves - are affected. 


\section{APPENDIX A}

Publications (10)

1. K.H. Burrell, T. Kurki-Suonio et al., 'Comparison of Thermal and Angular Momentum Transport in Neutral Beam-Heated Hot-Ion H- and L-mode Discharges in DIII-D', Proceedings of the 17th European Conference on Controlled Fusion and Plasma Physics (European Physical Society, Petit-Lancy, Switzerland, 1990), Vol. 14B, Part I, p.271, and General Atomics Report GA-A20058.

2. DIII-D Research Team (T. Kurki-Suonio as a UCB team member), 'Recent Results with Deuterium Plasma Operation of the DIII-D Tokamak', to be published in J. of Plasma Physics and Controlled Fusion.

3. J.L. Luxon et al. (T. Kurki-Suonio as a UCB team member), 'Recent Results from DIII-D and Their Implications for Next Generation Tokamaks', Plasma Physics and Controlled Fusion 32, 869 (1990).

4. M.A. Mahdavi et al. (T. Kurki-Suonio as a UCB team member), 'Plasma Boundary Experiments on DIII-D Tokamak', J. Nucl. Mater. 176, 32 (1990), and General Atomics report GA-A20130 (1990).

5. D.P. Schissel et al. (T. Kurki-Suonio as a UCB team member), 'The Effects of Carbonization on the Confinement Properties of the DIII-D H-Mode', Proceedings of the 17th European Conference on Controlled Fusion and Flasma Physics (European Physical Society, Petit-Lancy, Switzerland, 1990), Vol. 14B, Part I, p.275.

6. K.H. Burrell, T. Kurki-Suonio et al., 'Transport in Auxiliary-Heated, Hot-Ion H-Mode and L-Mode Discharges in the DIII-D Tokamak', General Atomics Report GAA20277, and Proceedings of the Thirteenth Intermational Conference on Plasma Physics and Controlled Nuclear Fusion Research Washington, D.C., 1990 (IAEA, Vienna, A ustria), Vol. 1, p. 123.

7. DIII-D Team (T. Kurki-Suonio as a UCB team member), 'DIII-D Research Program Progress', to be published in Plasma Physics and Controlled Nuclear Fusion Research 1990. (IAEA, Washington, DC, 1990).

8. A. Brizard, T.K. Fowler, D. Hua, and P.J. Morrison, 'Thermodynamic Constraints Applied to Tokamaks', to be publi-hed in Comments on Plasma Physics and Controlled Fusion, Vol. 14, No. 5.

9. D.D. Hua, X.Q. Xu, and T.K. Fowler, 'Ton-Temperature-Gradient Modes in NonCircular Tokamak Geometry', submitted for publication in Physics of Fluids B.

10. T.K. Kurki-Suonio, R.J. Groebner, and K.H. Burrell, 'Changes in Local Confinement after L-H Transition on DIII-D', General Atomics Report GA-A20450, and accepted for publication in Nuclear Fusion. 
Reports (9)

11. T. Kurki-Suonio and R. Groebner, 'Effect of ELMs on Ion Temperature Profile', DIII-D Physics Memo, D3DPM No. 9004

12. T.K. Fowler, and P.J. Morrison, 'Extremal Bounds on Drift Wave Growth Rates and Transport', UC-BFE-009, March 20, 1990.

13. D. Finkenthal, 'A Cookbook Guide to Learning The General Atomics CERFIT Code for Ion Temperature Analysis', UC-BFE-012, April 4, 1990.

14. Z. Covaliu, 'Sensitivity of a Tokamak Heat Transport Model to Edge Effects and Density profile', UC-BFE-013, June 18, 1990.

15. B. Modi, 'Speeding Up the CERFIT Code for Ion Temperature Analysis at DIII-D Tokamak', UC-BFE-019, 1991.

16. Q. Nguyen T.K. Fowler, and R.D. Stambaugh, 'Divertor Heat Flux Reduction Using Poloidal Coils', UC-BFE-020, 1991.

17. A. Brizard, 'Thermodynamic Analysis of Unstable Gyrokinetic Plasmas', UCBFE-021, 1991.

18. A. Brizard, D. Hua, and T.K. Fowler, 'Gyrokinetic Free Energy in Tokamak Plasmas', UC-BFE-022, March 7, 1991.

19. Joseph Fitzpatrick and T.K. Fowler,'Consequences of a Critical Gradient',UCBFE-026, November 3, 1991.

Talks, Posters (12)

20. T.K. Fowler, 'Thermodynamic Insights into Tokamak Confinement', poster at the Division of Plasma Physics meeting, APS, Hollywood, Florida, November 1988.

21. T.K. Fowler, and P.J. Morrison, 'Bounds on Instability Growth Rates in Tokamaks', Sherwood Theory Meeting, San Antonio, March 1989.

22. P.J. Morrison, and T.K. Fowler, 'Extremal Bounds on Instability Growth Rates', poster at the Division of Plasma Physics meeting, APS, Anaheim, California, November 1989.

23. G.D. Porter, D. Hill, M. Perry, and T. Kurki-Suonio, 'Transient Analysis of Particle Transport in DIII-D H-Mode Plasmas, (A talk given in The Transport Task Force Meeting, Hilton Head, South Carolina, Feb 17-23 1990)

24. D.F. Finkenthal, and T.K. Fowler, 'Effect of the Tae mode on Achieving Ignition in Tokamak Reactors', poster at the Division of Plasma Physics meeting, APS, Cincinnati, Ohio, 1990. 
25. D. Hua, A. Brizard, and T.K. Fowler, 'Gyrokinetic Free Energy in Tokamak Plasmas, poste $i$ at the Division of Plasma Physics meeting, APS, Cincinnati, Ohio, 1990.

26. Q. Nguyen, T.K. Fowler, and R.D. Stambaugh, 'Flux Expansion at the Divertor Plates', poster at the Division of Plasma Physics meeting, APS, Cincinnati, Ohio, 1990.

27. T. Kurki-Suonio and R. Groebner, Changes in Local Confinement after an L-H Transition in DIII-D', poster at the Division of Plasma Physics meeting, APS, Cincinnati, Ohio, 1990.

28. D. Finkenthal, T.K. Fowler, K. Burrell, W.P. West, R. Seraydarian, J. Kim, and P. Gohil, 'Helium Density Profile Measurements on the DIII-D Tokamak Using Charge Exchange Recombination (CER) Spectroscopy', poster at the Division of Plasma Physics meeting, APS, Tampa, Florida, 1991.

29. D. Hua, X. Xu, and T.K. Fowler, 'Ion-Temperature-Gradient Modes in NonCircular Geometry', poster at the Division of Plasma Physics meeting, APS, Tampa, Florida, 1991.

30. T. Kurki-Suonio, R.J. Groebner, R. Philipona, C. Rettig, E. Doyle, K.H. Burrell, and W.A. Peebles, 'Comparison of Reduction in Fluctuations and Transport on DIII-D', poster at the Division of Plasma Physics meeting, APS, Tampa, Florida, 1991.

31. W.A. Peebles, R. Philipona, T. Kurki-Suonio, et al., 'Turbulence Reduction and Improved Confinement in DIII-D H-mode Plasmas', oral contributed paper at the Division of Plasma Physics meeting, APS, Tampa, Florida, 1991.

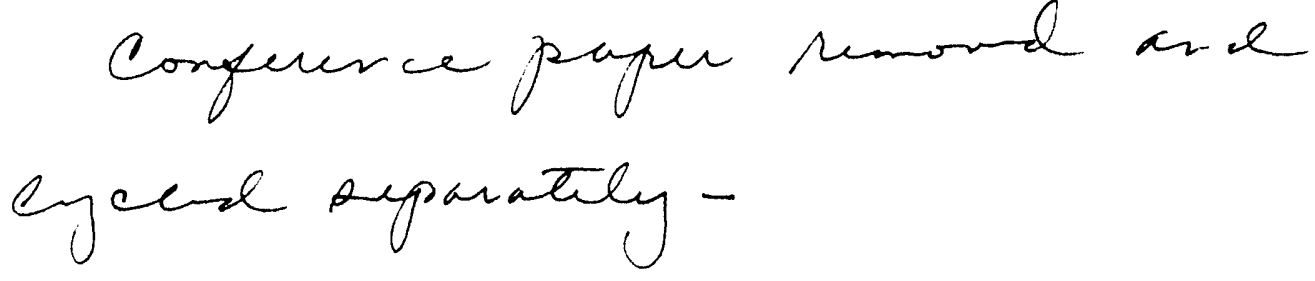



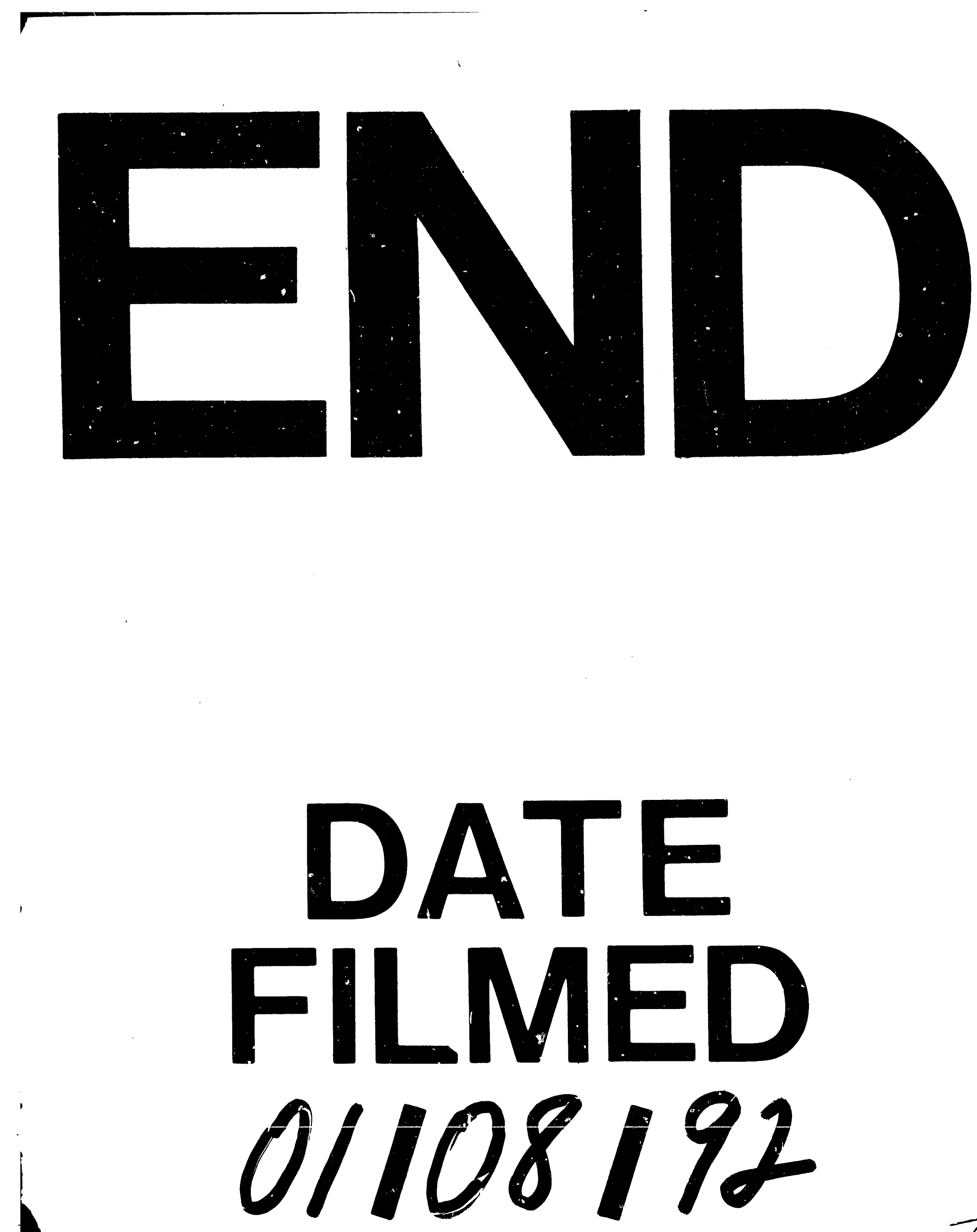
